\title{
DYNAMICS OF DROPLET MOTION INDUCED BY ELECTROWETTING
}

\author{
Yi Lu ${ }^{1}$, Aritra Sur ${ }^{1}$, Carmen Pascente ${ }^{2}$, S. Ravi Annapragada ${ }^{3}$, \\ Paul Ruchhoeft ${ }^{2}$, and Dong Liu ${ }^{1, \S}$
}

\begin{abstract}
Electrowetting (EW) has drawn significant interests due to the potential applications in electronic displays, lab-on-a-chip microfluidic devices and electro-optical switches, etc. However, current understanding of EW is hindered by the inadequacy of available numerical and theoretical methods in properly modeling the transient behaviors of EW-actuated droplets. In the present work, a combined numerical and experimental approach was employed to study the EW response of a droplet subject to both direct current (DC) and alternating current (AC) actuating signals. Computational fluid dynamics models were developed by using the Volume of Fluid (VOF)-Continuous Surface Force (CSF) method. A dynamic contact angle model based on the molecular kinetic theory was implemented as the boundary condition at the moving contact line, which considers the effects of the contact line friction and the pinning force. The droplet shape evolution under DC condition and the interfacial resonance oscillation under $\mathrm{AC}$ condition were investigated. It was found that the numerical models were able to accurately predict the key parameters of electrowetting-induced droplet dynamics.
\end{abstract}




\section{INTRODUCTION}

Droplet spreading and oscillation on a solid substrate has attracted great research interest due to its relevance in a variety of engineering applications, such as droplet-based microfluidics [1], optofluidic optical attenuators [2,3] and reflective displays [4], etc. Among various approaches to control the droplet motion, electrowetting (EW) is particularly convenient and versatile for its ability to tune the surface wetting property with an external electric field. With EW, it is now possible to design digitized active and reconfigurable cooling devices for high-flux thermal management of compact microsystems [5-8]. EW originates from the electrical force concentrated at the three-phase contact line (TCL), which causes the apparent contact angle, $\theta_{a p p}$, to deviate from the inherent equilibrium value, $\theta_{e}$. The EW-induced contact angle variation can be described by the Young-Lippmann equation

$$
\cos \theta_{a p p}=\cos \theta_{e}+\frac{\varepsilon_{0} \varepsilon_{r}}{2 d \sigma_{l v}} U^{2}
$$

where $\varepsilon_{0}$ is the vacuum permittivity constant, $\varepsilon_{r}$ the relative permittivity of the insulating dielectric layer, $d$ the thickness of the dielectric layer, $\sigma_{l v}$ the surface tension of the liquid-vapor interface, and $U$ the applied voltage.

As the foundation of EW theory, Eq. (1) has been widely used to predict the EW response of liquid droplets [9-11]. It is expected to hold as long as the applied voltage is below the thresholds for contact angle saturation (i.e., the contact angle reaches a limiting value irrespective of further increase in voltage) and edge instability (i.e., satellite droplets are disintegrated from the mother droplet) [1]. However, the resulting $\theta_{\text {app }}$ only accounts for the final state of a droplet after it reaches the equilibrium. It has been long known that the static contact angle (SCA) concept is inadequate in dealing with the transient processes, where the droplet deforms as a function of time and $\theta_{a p p}$ varies between the limits set by the advancing and receding contact angles $[12,13]$. In EW, $\theta_{a p p}$ is affected by the interplay of the inertial, viscous, capillary, electrical and contact line friction forces, and may deviate significantly from the static prediction by Eq. (1) $[14,15]$. Moreover, both direct current (DC) and alternating current (AC) signals can be applied to induce EW (referred to DCEW and ACEW, respectively, in this paper). The temporal evolution of a droplet in ACEW also depends on the relative magnitudes of the natural resonant frequency of the droplet, $f_{N}$, the driving frequency of the electric field, $f$, the charge relaxation frequency, $f_{r}$

$$
f_{r}=\sigma / \varepsilon_{0} \varepsilon_{r}
$$

and the crossover frequency, $f_{c}$

$$
f_{c}=\frac{\sigma}{\left[\varepsilon_{f}+\varepsilon_{r}\left(\frac{R_{d}}{d}\right)\right]}
$$


where $\sigma$ and $\varepsilon_{f}$ are the electrical conductivity and the relative permittivity of the fluid, and $\mathrm{R}_{\mathrm{d}}$ the characteristic size of the droplet. Therefore, a dynamic contact angle (DCA) model, rather than Eq. (1), must be employed to fully characterize the droplet motion under electrowetting actuation.

Historically, both the hydrodynamic theory $[16,17]$ and the molecular kinetic theory (MKT) [18] have been developed to model the DCA of a droplet that is displacing on a solid surface. The hydrodynamic theory attributes the deviation of the DCA from SCA to bulk viscous dissipation in the liquid front near the moving contact line, whereas the MKT takes into account the friction force owing to the adsorption or desorption of fluid particles at the immediate vicinity of the contact line [19]. Past studies suggest the MKT is more suitable for the study of EW as it can be applied to a wider range of contact line velocities $\left(3.16 \times 10^{-5}-10 \mathrm{~m} / \mathrm{s}\right)$ [20] and offers a straightforward explanation of the contact angle hysteresis phenomenon, thereby yielding more accurate predictions of the droplet dynamics [21-23]. Recently, a few experimental and numerical studies have successfully demonstrated the validity of MKT in DCEW applications [14, 15, 24-28]. In particular, Keshavarz-Motamed et al. [25] implemented a MKT-based DCA model in the numerical simulation of droplet displacement in a parallel plate microchannel. The results revealed that the dynamic features of wetting must be considered, or the simulation will overestimate the effects of EW actuation, including the contact angle, aspect ratio and velocity of the droplet. By combining MKT with an idealized drop geometry, Annapragada et al. [26] investigated the EW response of a droplet to a DC step input signal, and formulated a kinematic equation for the time-dependent spreading of the drop on the substrate, which correctly predicted the overall trend of the contact line motion.

The utility of DCA models in ACEW analysis is further complicated by the presence of timeharmonic shape oscillations at the free surface of the droplet [29-31]. The basic assumptions commonly adopted for DCEW, such as a quasi-steady state, the spherical cap shape of the droplet and the negligence of inertia, are no longer valid for ACEW. Consequently, most available theoretical and numerical studies of ACEW chose to circumvent the complexities involving DCA. Oh et al. [29] conducted a theoretical analysis of interfacial oscillations of a droplet in ACEW by applying a domain perturbation method. The oscillating droplet was regarded as a half-region of a spherical drop in an unbounded domain, and its shape was represented by a linear combination of an infinite number of shape modes. This model was able to qualitatively reproduce the resonance modes observed in the experiments, but, since the capillary force and the contact line friction were completely omitted, it was only valid for weak viscous effect and small drop deformations. Ashoke Raman et al. [32] numerically investigated the dynamics of ACEWinduced droplet jumping by the means of a high-density ratio based lattice Boltzmann method (LBM). The SCA model (Eq. (1)) was used together with a geometric formulation to describe the wetting boundary condition. The findings revealed some interesting features of the droplet lift-off and transport 
mechanism, however, no experimental validation was provided. Hong et al. [33] employed an effective electrical wetting tension at the contact line to model the effect of electric field on the droplet wetting behavior. By treating the ACEW response as a quasi-electrostatic problem, they analyzed the AC electric field around the droplet and computed the electrical wetting tension from the integration of the Maxwell stress. The results were then applied in a varied form of Eq. (1) to acquire the time-averaged contact angle at different actuating frequencies. In a study of droplet dynamics in ACEW, Li et al. [34] followed the electrical wetting tension concept but used a dissipative particle dynamics (DPD) approach, which is a modified version of the lattice gas method by replacing single fluid molecules with coarse-grained particle clusters. Unfortunately, only the oscillations of a sub-micrometer droplet in ACEW were explored due to the computational limits of the DPD approach. Recently, Li et al. [35] presented the first numerical simulation of droplet oscillation in ACEW that incorporated a DCA model to describe the contact line motion. They used an axisymmetric model, in conjunction with the moving mesh interface tracking (MMIT) method, for solving the Navier-Stokes equations. This study focused on the resonance phenomenon and the oscillation patterns at different actuating frequencies.

Gaining a fundamental understanding of the droplet dynamics is crucial to the performance prediction and design of current and future EW-based devices. The literature survey reveals that the available numerical and theoretical methods are inadequate in accurately simulating the complex electrohydrodynamic transport associated with EW, especially ACEW. Thus, it is the aim of the present study to overcome the deficiencies of existing numerical models and to provide more in-depth insights into the dynamics of EW-induced droplet motion. The rest of the paper is organized as follows. First, the elementary DCA models from the hydrodynamic theory and MKT are briefly reviewed. Then, the experimental setup for both DCEW and ACEW tests is presented. Subsequently, the numerical methods using VOF method are presented in detail. At last, the numerical predictions are validated with the experimental measurements and the key characteristics of the EW-induced droplet dynamics are discussed.

\section{THEORETICAL BACKGROUND}

\section{Dynamic contact angle in electrowetting}

The dynamics of droplet motion can be quantified by the dynamic contact angle, $\theta_{d}$, and the contact line velocity, $u_{c l}$, i.e., the velocity at which the liquid front moves across the solid surface. Hence, a certain relation is expected between $\theta_{d}$ and $u_{c l}$ for a given system. Classical hydrodynamic models attribute the observed DCA to viscous bending of the liquid-vapor interface at a mesoscopic region near the contact line. The bulk viscous dissipation is assumed to be the dominant resistance to the contact line motion. However, solving the flow field alone does not yield a physically meaningful solution because a 
conceptual conflict exists between a moving contact line and the no-slip boundary condition at the solid wall, which predicts an unbounded stress at the contact line and an infinite force on the wall. To eliminate the singularity, hydrodynamic models separate the liquid into an outer region, where the conventional no-ship boundary is still applicable, and an inner region, where the continuum description breaks down and the fluid slippage occurs in the first couple of layers of liquid molecules adjacent to the wall. The resulting correlation describes the DCA in terms of the capillary number, $\mathrm{Ca}$, [36, 37],

$$
\theta_{d}^{3}-\theta_{e}^{3}= \pm 9 C a \ln \left(\frac{L^{\prime}}{L_{m}}\right)
$$

where $C a=\mu_{l} u_{c l} / \sigma_{l v}$ and $\mu_{l}$ is the liquid viscosity. In Eq. (4), $L^{\prime}$ and $L_{m}$ are the macroscopic and microscopic length scales for the outer and inner regions, respectively, and the plus sign applies to an advancing liquid front whereas the minus sign applies to a receding liquid movement. For small $\mathrm{Ca}$, Eq. (4) can be approximated by the Hoffman-Voinov-Tanner law [12, 38, 39],

$$
\theta_{d}^{3}-\theta_{e}^{3}=C_{T} C a
$$

where $C_{T}$ is a numerical constant $\left(C_{T} \approx 72\right)$. In contrast, by adopting the Frenkel/Eyring view of liquid transport [40,41], the MKT treats the contact line motion as a series of adsorption and desorption events of liquid molecules on the solid wall. Thus, the energy dissipation is not due to the bulk viscous flow, but rather the result of the attachment and detachment of fluid particles at the immediate vicinity of the contact line. In this theory, the velocity of the contact line is determined by the equilibrium frequency of the random molecular displacement, $k^{0}$, and the average length of each displacement, $\lambda$. Thus, the velocity-dependence of the DCA is attributed to the disturbance of adsorption equilibria and the subsequent out-of-balance surface tension, $F_{w}=\sigma_{l v}\left(\cos \theta_{e}-\cos \theta_{d}\right)$, acting as the driving force. The subsequent equation for the contact line velocity is

$$
u_{c l}=2 k^{0} \lambda \sinh \left[\sigma_{l v}\left(\cos \theta_{e}-\cos \theta_{d}\right) \lambda^{2} / 2 k_{B} T\right]
$$

where $k_{B}$ is the Boltzmann constant and $T$ the absolute temperature. If the value of the sinh function is sufficiently small, Eq. (6) reduces to

$$
u_{c l} \approx \frac{k^{0} \lambda^{3} \sigma_{l v}\left(\cos \theta_{e}-\cos \theta_{d}\right)}{k_{B} T}=\sigma_{l v}\left(\cos \theta_{e}-\cos \theta_{d}\right) / \varsigma
$$

where $\varsigma=k_{B} T / k^{0} \lambda^{3}$ is called the coefficient of contact line friction. The physical implication of Eq. (7) becomes more evident if it is recast in the form of balance of forces at the contact line

$$
\sigma_{l v} \cos \theta_{d}=F_{c}+F_{c l}
$$

where the capillary force is represented by $F_{c}=\sigma_{l v} \cos \theta_{e}$ and the contact line friction force is $F_{c l}=$ $-s u_{c l}$.

In electrowetting, the contact line is made to move by the induced electrical force. To represent this effect, the force balance equation is modified as [23]

$$
\sigma_{l v} \cos \theta_{d}=F_{E W}+F_{c}+F_{c l}
$$


where $F_{E W}$ is the electrical force

$$
F_{E W}=\frac{\epsilon_{0} \epsilon_{r} U^{2}}{2 d}
$$

It is noted that, if the actuating frequency $f$ exceeds $f_{c}$ (given by Eq. (3)), the frequency-dependent electrical properties must be considered when solving for the electrowetting force [1, 33]. As will be shown later, $f_{c}$ is estimated to be $2.4 \mathrm{kHz}$ in this study, one order of magnitude higher than $f$, which varies between 0 and $200 \mathrm{~Hz}$. Thus, the droplet is expected to behave like a conductor, and Eq. (10) offers an adequate description of the electrical force for both DCEW and ACEW in the present work. Further, considering the pinned edge model [42], a pinning force term, $F_{p i n}$, is added to the expression of $F_{c l}$, which represents the maximum force that can resist the motion of the droplet

$$
F_{p i n}=c_{p i n} \operatorname{sgn}\left(u_{c l}\right)-\frac{c_{p i n}}{\pi / 2} \tanh ^{-1}\left[\frac{\varsigma u_{c l}}{c_{p i n} /(\pi / 2)}\right]
$$

where $c_{p i n}$ is determined from the contact angle hysteresis effect [23]. Equation (11) indicates the pinning force attains its maximum, $c_{p i n} \operatorname{sgn}\left(u_{c l}\right)$, when the contact line is about to move, and vanishes if $u_{c l}$ becomes sufficiently high. Accordingly, the friction force becomes

$$
F_{c l}=-\left\{\varsigma u_{c l}+c_{p i n} \operatorname{sgn}\left(u_{c l}\right)-\frac{c_{p i n}}{\pi / 2} \tanh ^{-1}\left[\frac{\varsigma u_{c l}}{c_{p i n} /(\pi / 2)}\right]\right\}
$$

Combining Eqs. (9), (10) and (12), the following DCA relationship is developed

$$
\theta_{d}=\cos ^{-1}\left\{\cos \theta_{e}+\frac{\epsilon_{0} \epsilon_{r}}{2 d \sigma_{l v}} U^{2}-\left[\varsigma u_{c l}+c_{p i n} \operatorname{sgn}\left(u_{c l}\right)-\frac{c_{p i n}}{\pi / 2} \tanh ^{-1}\left[\frac{\varsigma u_{c l}}{c_{p i n} /(\pi / 2)}\right]\right] / \sigma_{l v}\right\}
$$

This DCA model will be applied as a key boundary condition in the numerical simulations of EW.

\section{EXPERIMETAL SETUP}

The experimental apparatus for the electrowetting tests is shown in Fig. 1. It consisted of the test piece, the control circuit and the imaging system. The test piece was made of a 3" silicon wafer (Silicon Quest) with a 500-nm-thick, thermally grown silicon dioxide $\left(\mathrm{SiO}_{2}\right)$ layer $\left(\varepsilon_{r}=3.9\right)$. A thin layer $(70$ $\mathrm{nm}$ ) of Teflon (AF2400, Dupont) was spin-coated on the wafer to produce the hydrophobic surface. To improve the adhesion of Teflon to $\mathrm{SiO}_{2}$, a silane-based adhesion promoter (FSM-660-4, Cytonix) was dip-coated on $\mathrm{SiO}_{2}$ before the spin-coating procedure. Since silicon has a reasonable electrical conductivity $\left(1.56 \times 10^{-3} \mathrm{~S} / \mathrm{m}\right.$ at $\left.20^{\circ} \mathrm{C}\right)$, the substrate works directly as the ground electrode. Before the experiment, a $9.0 \pm 0.1 \mu \mathrm{L}$ deionized water droplet was dispensed gently onto the test piece with a micropipette. A $99.99 \%$ pure platinum wire of a $100-\mu \mathrm{m}$ diameter was inserted into the droplet from the top as the actuating electrode (as shown in Fig. 1). Since the experiments were conducted at room temperature and each test only lasted for a few seconds, the effect of evaporation was negligible and the droplet volume was assumed constant. The properties of water are: electrical conductivity $\sigma=2 \times 10^{-4}$ $\mathrm{S} / \mathrm{m}$, relative permittivity $\varepsilon_{f}=88$, density $\rho_{l}=998 \mathrm{~kg} / \mathrm{m}^{3}$, viscosity $\mu_{l}=1.002 \times 10^{-3} \mathrm{~Pa} \bullet \mathrm{s}$, and surface 
tension $\sigma_{l v}=0.0728 \mathrm{~N} / \mathrm{m}$. For a characteristic droplet size $R_{d}=1.18 \mathrm{~mm}$, Eq. (3) yields a crossover frequency of $f_{c} \approx 2.4 \mathrm{kHz}$.

The actuation signals for EW were provided by an arbitrary waveform generator (Fluke 294-U, Fluke) in combination with an inverting power amplifier (BOP 200-1D BIT 4886, KEPCO). A stepfunction signal was used for DCEW tests, and sinusoidal functions of various frequencies were applied to actuate ACEW. The EW response of the droplet was recorded at 6,000-10,000 frame per second (fps) using a high-speed video camera (FASTCAM Ultima APX, Photron). The shutter speed was set to $1 / 16000 \mathrm{~s}$, and a cold light illumination source was used to compensate for the short exposure time at high frame rates. A Nikon 18-105 mm lens (f 3-5.6) was employed to observe the details of the droplet motion. The pixel resolution ranges from $17.1 \mu \mathrm{m}$ to $33.5 \mu \mathrm{m}$, depending on the distance from the droplet to the lens. The control circuit and the imaging system were synchronized using a pulse generator (BNC 565, Berkeley Nucleonics).

Once the droplet motion was recorded, the stream of static images were extracted from the video and analyzed using an in-house image-processing program developed in MATLAB. The measurements included the instantaneous contact angle $\left(\theta_{d}\right)$, the contact radius $(R)$ of the wetted spot on the wall and the height of the droplet. The experimental uncertainties in the contact angle and contact radius/height measurements are $\pm 2^{\circ}$ and $\pm 0.02 \mathrm{~mm}$, respectively. A subpixel smoothing method and a high-order polynomial fitting algorithm [43] were employed to precisely identify the droplet profile and the contact points of the droplet with the solid surface. In particular, the polar coordinate system was used to fit the droplet profile with a fourth order polynomial. The tangent to the droplet profile at the contact point was used to determine the CA. The intrinsic CA of a water droplet on the Teflon surface was measured to be $118^{\circ} \pm 2^{\circ}$ and the initial contact radius was $\mathrm{R}_{0}=1.18 \pm 0.02 \mathrm{~mm}$.

\section{NUMERICAL METHODS}

Numerous studies have demonstrated the effectiveness of the volume of fluid (VOF) method in predicting the droplet motion on a solid surface [44-48]. In the current work, the VOF-Continuum Surface Force (CSF) model was used in the commercial computational fluid dynamics (CFD) software package, FLUENT, to investigate the transient droplet dynamics under the influence of DCEW and ACEW. Special attention was given to take care of two main challenges: (1) the prescription of the boundary condition at the moving contact line, and (2) the incorporation of the DCA model in the computation. 


\section{VOF-SCF model}

The VOF model tracks the time-dependent deformation of the interface by computing the distribution of the volume fraction of two immiscible fluids in the computational domain. The volume fraction of a secondary phase, $\alpha_{s}$, is obtained by solving the continuity equation

$$
\partial \alpha_{s} / \partial t+\nabla\left(\alpha_{s} \vec{u}\right)=0
$$

The volume fraction of the primary phase, $\alpha_{p}$, can be computed from

$$
\alpha_{p}=1-\alpha_{s}
$$

Here, $\alpha_{s}=1$ represents a computational cell that is completely occupied by the gas phase, and $\alpha_{s}=0.5$ is taken to be the location of the liquid-gas interface. The thermophysical properties in a single cell, such as the density and viscosity, are calculated as the volume average

$$
\begin{aligned}
& \rho=\alpha_{s} \rho_{s}+\alpha_{p} \rho_{p} \\
& \mu=\alpha_{s} \mu_{s}+\alpha_{p} \mu_{p}
\end{aligned}
$$

A single set of Navier-Stokes equations is solved for the average velocity of the mixture, which is shared by all the phases

$$
\frac{\partial}{\partial t}(\rho \vec{u})+\nabla(\rho \vec{u} \vec{u})=-\nabla p+\nabla \cdot\left[\mu\left(\nabla \vec{u}+\nabla \vec{u}^{T}\right)\right]+\rho \vec{g}+\vec{F}
$$

where p, $\overrightarrow{\boldsymbol{g}}$ and $\overrightarrow{\boldsymbol{F}}$ are the pressure, gravitational acceleration and body force, respectively. For computational cells that are not at the liquid-vapor interface, only one phase is present and just $\vec{g}$ needs to be considered. For the interfacial cells, instead of treating the surface tension as a pressure jump boundary condition across the interface, an equivalent volumetric body force $\vec{F}$ is considered using the CSF formulation [49]. For a two-phases system, $\vec{F}$ is given as

$$
\vec{F}=\sigma_{l v} \frac{2 \rho \kappa_{s} \nabla \alpha_{s}}{\left(\rho_{p}+\rho_{s}\right)}
$$

where $\kappa_{\mathrm{s}}$ is the interfacial curvature for the second phase, and $\vec{n}$ is the unit vector normal to the interface

$$
\begin{aligned}
& \kappa_{s}=-(\nabla \cdot \vec{n}) \\
& \vec{n}=\nabla \alpha_{s} /\left|\nabla \alpha_{s}\right|
\end{aligned}
$$

The interfacial shape at the contact line is imposed by specifying the unit normal using the contact angle

$$
\vec{n}=\vec{n}_{w} \cos \left(\theta_{w}\right)+\vec{t} \sin \left(\theta_{w}\right)
$$

where $\hat{n}_{w}$ and $\hat{t}_{w}$ are the unit vectors normal and tangential to the wall, which are directed into the fluid and the wall, respectively. 


\section{Computational model}

The computational domain is illustrated in Fig. 2, where a truncated spherical drop of water (shown as the shaded black) is patched at the corner of the domain. The initial contact angle and contact radius were $118^{\circ}$ and $1.18 \mathrm{~mm}$, respectively. The shape change owing to the insertion of the electrode from the top of the droplet was neglected. Due to the axisymmetric nature of the drop geometry, a 2D axisymmetric VOF model was developed in FLUENT. A no-slip boundary condition was specified at the bottom wall. The upper and right boundaries were set as at ambient pressure $\left(P=P_{a t m}\right)$. A rather large domain size of $24 \mathrm{~mm} \times 24 \mathrm{~mm}$ was chosen in order to eliminate the effect of boundary-induced disturbances.

\section{DCA implementation in VOF}

In the VOF-CSF simulations, the MKT-based DCA model was applied as a boundary condition at the contact line via user-defined functions (UDFs). According to Eq. (13), the required inputs include the applied voltage $U(t)$, the contact line velocity $u_{c l}(t)$, the frictional coefficient $\zeta$, and the pinning force $c_{p i n}$.

A step input voltage was used to actuate DCEW, and sinusoidal signals with various driving frequencies $(f)$ were used for ACEW

$$
U(t)=U_{0} \sin (2 \pi f t)
$$

In the following discussion, the AC signal will be reported in terms of its root mean square (RMS) value, i.e., $U_{R M S}=U_{0} / \sqrt{2}$.

Obtaining a rigorous estimate of the velocity of the contact line, $u_{c l}(t)$, is challenging, due to the singularity dilemma at the contact line $[22,45,50]$. If the no-slip condition is specified on the solid wall, the contact line cannot move. However, the contact line does move in reality, thereby leading to an infinite viscous shear and a diverging drag force on the wall. To avoid this, a Navier-slip boundary condition, $u_{\text {slip }}=\lambda \partial u / \partial y$, can be used to represent the contact line motion as a slip, where $\lambda$ is the slip length, i.e., the distance from the boundary where a linearly extrapolated velocity profile would reach zero. Unfortunately, the actual slip length ( $\sim$ tens of nanometers) is much smaller than the mesh size in most numerical simulations, and the available computational resources do not allow the slip velocity to be accurately resolved $[44,51]$. On the other hand, the VOF implementation uses the cell face normal velocity to advect the volume fraction, thus an implicit ("effective") slip length of one mesh size is included by default, even though the no-slip condition is enforced on the solid wall [52]. Consequently, the contact line velocity computed by the VOF methodology is mesh-dependent: a coarser mesh leads to a larger slip length and, therefore, a larger contact line velocity, and vice versa. However, it is seen from Eqs. (9) to (13) that an increase in the contact line velocity results in a larger dynamic contact angle, and 
Afkhami et al. [52] showed larger contact angles allow the surface tension force to balance the stress singularity, thus slowing the moving contact line. As a result, the overall contact line motion becomes largely insensitive to the mesh spacing $[25,26,47,51,53]$. Following this observation, $u_{c l}(t)$ in the present work is taken as the normal velocity of the liquid-gas interface at one half-cell height above the solid wall. It is noted that an estimate of $u_{c l}$ can be also calculated by numerically differentiating the interfacial contact radius: $u_{c l}(t)=\frac{d R}{d t}[48,54]$. However, the result obtained from this approach is highly sensitive to the time step used. Hence, it was not used in the present work.

The friction coefficient, $\zeta$, can be determined experimentally by fitting the measured DCA data with a certain DCA model. However, the value differs in the available reports. In general, it was found that a larger value $\left(\zeta=0.2-0.4 \mathrm{Ns} / \mathrm{m}^{2}\right)$ fit the experimental data well when the contact angle hysteresis effect is not considered [14, 24, 26], otherwise a smaller value $\left(\zeta=0.18 \mathrm{Ns} / \mathrm{m}^{2}\right)$ is more suitable $[23,30]$. In the simulations of this work, $\zeta=0.15 \mathrm{Ns} / \mathrm{m}^{2}$ is used as it yields more accurate predictions of the experimental data.

The pinning force, $c_{p i n}$, is deduced from the contact angle hysteresis [23] as

$$
c_{p i n} \approx \sigma_{l v}\left|\cos \theta_{e}-\cos \theta_{a}\right|
$$

or

$$
c_{p i n} \approx \sigma_{l v}\left|\cos \theta_{e}-\cos \theta_{r}\right|
$$

where $\theta_{a}$ and $\theta_{r}$ are the advancing and receding contact angles, respectively. The experimental results showed the maximum contact angle hysteresis $\left(\theta_{a}-\theta_{r}\right)$ is about $16^{\circ}$. Hence, $c_{p i n}$ is set as $0.009 \mathrm{~N} / \mathrm{m}$ in the simulations, which corresponds to $\left|\theta_{a}-\theta_{e}\right|=8^{\circ}$.

\section{Mesh Independence}

Three different mesh sizes, $200 \times 200,267 \times 267$ and $400 \times 400$, were employed in this study, which correspond to 10,13 and 20 grids per initial contact radius of the droplet, respectively. Figure 3 illustrates the predicted time evolution of the contact radius of the droplet as compared to the experimental measurements (Note: the contact radius was normalized by the initial contact radius, $\mathrm{R}_{0}$ ). The results show that the $200 \times 200$ mesh yields a satisfactory prediction of the measurements and further mesh refinement does not drastically improve the prediction accuracy. This is consistent with the findings in $[44,47]$ that a resolution of 10 grids per drop radius is sufficient to capture the dynamics of the droplet motion. Hence, a mesh size of $200 \times 200$ was used in all the simulations of this work. 


\section{RESULTS AND DISCUSSION}

\section{Droplet dynamics in DCEW}

Figure 4 shows the comparison of the experimental results (left) with the VOF simulation predictions (right) of the instantaneous droplet shape at different time instants for a step input voltage ( $V$ $=32 \mathrm{~V})$. It is observed that the droplet remains a spherical cap shape before the signal is applied $(t=0$ $\mathrm{ms}$ ). Once the voltage is turned on, the contact line moves outward. The shape of the droplet distorts as the electrical force reduces the contact angle from $\theta_{e}$ to the new equilibrium value $\theta_{a p p}$. The detailed droplet dynamics can be visualized through the internal flow field within the droplet, as shown in Fig. 5. Clearly, the droplet motion originates from the contact line at $t=0.1 \mathrm{~ms}$. The high velocity region represents the commencement of capillary waves, and the rest of the fluid inside the droplet remains stationary. As time elapses, the capillary waves propagate upward along the liquid-gas interface, causing the impacted part of the droplet to deform continuously $(t=0.5-3.5 \mathrm{~ms})$. At $t=4 \mathrm{~ms}$, the wave fronts reach the apex and the focusing effect causes the droplet height to rise momentarily. Subsequently, the surface waves are reflected back to the bottom of the droplet, thereby lowering the height and further spreading the contact radius on the wall $(t=6-20 \mathrm{~ms})$.

The time history of the normalized contact radius, normalized height and instantaneous contact angle of the droplet is plotted in Fig. 6 for a pulse wave input ( $V=32 \mathrm{~V}$ and the duty cycle $T_{0}=500 \mathrm{~ms}$ ). The height is normalized relative to the initial height of the droplet. The results show that the contact line first expands on the wall and, after reaching the maximum radius at $t=11 \mathrm{~ms}$, it recoils and undergoes damped oscillations until a new equilibrium is established at $t=15 \sim 16 \mathrm{~ms}$ (The final normalized contact radius is about 1.35). The overshoot in contact radius is the consequence of the inertia of the internal flow induced by the contact line motion. After the voltage returns to zero ( $t=500 \mathrm{~ms})$, the contact line rapidly contracts to a minimum radius $(t=512 \mathrm{~ms})$ before restoring to a final size, which is slightly larger than the initial radius due to the contact angle hysteresis effect. The time constants for the droplet expansion and contraction processes are found to be roughly the same, 15 16 ms. As compared to the short transient in contact radius, Fig. 6 depicts a sustained oscillating behavior in the height evolution that barely reaches an equilibrium in the duration of the experiment. This can be attributed to the different damping mechanisms for the contact radius and the height [26]: the motion of the contact line is dampened by the contact line friction, whereas the height becomes stabilized only after the inner flow is completely subdued by the much slower viscous dissipation. Another observation in Fig. 6 is that the contact angle is out of phase with the height during the oscillation, i.e., an increasing contact angle is always accompanied by a decrease in the height of the droplet, as required by the constant volume constraint. 


\section{Droplet dynamics in ACEW}

Shape oscillations of the droplet in ACEW were investigated with sinusoidal input voltages of 32 $\mathrm{V}_{\text {RMS }}$. The frequency of the actuating signal, $f$, varies from 0 to $200 \mathrm{~Hz}$. Figure 7 compares the simulation predictions (left) with the experimental results (right) of the instantaneous droplet shape at different time instants for three different actuating frequencies, $f=27 \mathrm{~Hz}, 79 \mathrm{~Hz}$ and $150 \mathrm{~Hz}$, respectively. Resonance oscillations of the interface can be observed clearly from the images. It is interesting to note that the oscillating frequencies of the droplet $\left(f_{\text {exp }}\right)$ are $50 \mathrm{~Hz}, 156 \mathrm{~Hz}$ and $278 \mathrm{~Hz}$, about twice the corresponding signal frequencies. This is because the droplet oscillation originates from the cyclic motion of the contact line, which is driven by the electrical force that is proportional to the square of the input voltage (as indicated by Eq. (10)). The resonance frequency of a free, inviscid droplet, $f_{n}$, is given by [55]

$$
f_{n}=\frac{1}{2 \pi}\left[n(n-1)(n+2) \frac{\sigma_{l v}}{\rho_{l} L^{3}}\right]^{1 / 2}
$$

where $\rho_{l}$ is the liquid density, $L$ is the volume-average radius of the droplet, and $n$ is an integer corresponding to different oscillation modes $(n=1,2, \ldots)$. Table 1 enumerates the actuating frequency, $f$, the measured oscillating frequency, $f_{\text {exp }}$, and the resonance frequency, $f_{n}$, of a $9 \mu \mathrm{L}$ droplet. It is seen $f_{\text {exp }}$ deviates from $f_{n}$ by $10-13 \%$, and this can be attributed to the viscous damping due to the presence of a moving contact line and the viscous boundary layer at the droplet-substrate interface. Hence, the droplet is oscillating at (or near) its resonance frequencies, and the shape patterns in Fig. 7 correspond to three resonance modes, $\mathrm{P}_{2}, \mathrm{P}_{4}$ and $\mathrm{P}_{6}$, respectively.

The shape modes of the droplet oscillations are better resolved in Fig. 8. Each image is the superimposition of over 100 images recorded by the high-speed camera during at least one oscillation cycle. The black arrows denote the node points where the local displacement of the free surface is always zero. It is observed that, as the mode number increases, the number of nodes and lobes also increases, whereas the oscillation amplitude (i.e., the lobe size) becomes smaller. For instance, $\mathrm{P}_{2}$ mode occurs at $f$ $=27 \mathrm{~Hz}$ and is characterized by the oblate and prolate spheroidal shapes with two nodes and three lobes. Similarly, the $\mathrm{P}_{4}$ mode takes place at $79 \mathrm{~Hz}$ with four nodes and five lobes, and the $\mathrm{P}_{6}$ mode at $150 \mathrm{~Hz}$ with six nodes and seven lobes. In the experiments, it is possible to identify other higher-order shape modes, such as $\mathrm{P}_{8}$ and $\mathrm{P}_{10}$, etc. However, the size of the lobes decreases drastically with increasing mode numbers, making them difficult to distinguish.

Figure 9 shows the oscillation amplitudes (represented by the maximum normalized contact radius and height) as a function of the actuating frequency $f$. It is observed that the oscillation almost vanishes at high frequencies $(f \rightarrow 200 \mathrm{~Hz})$. Local maxima of descending amplitudes can be found at three eigenfrequencies, $f=27 \mathrm{~Hz}, 79 \mathrm{~Hz}$ and $150 \mathrm{~Hz}$, respectively. This observation can be explained as 
follows. In EW, four resistive forces, including the inertia $\left(F_{i}\right)$, the viscous force $\left(F_{v}\right)$, the capillary force $\left(F_{c}\right)$ and the contact line friction force $\left(F_{c l}\right)$, act against the droplet deformation induced by the electrical force $\left(F_{E W}\right)$. Using $\mathcal{L}=2 R_{0}, T=\frac{1}{2 \pi f_{n}}$ and $u=R_{0} / T$ as the characteristic scales of length, time and velocity, these forces (per length) can be estimated as $F_{i} \sim \rho_{l} u^{2} L, F_{v} \sim \mu_{l} u, F_{c} \sim \sigma_{l v}$ and $F_{c l} \sim \varsigma u$. The frequencydependence of all five forces is plotted in Fig. 10. It shows that, while the capillary force remains comparable to the electrical force, the inertia and the contact line friction become dominant as the frequency increases. At sufficiently high frequencies, they will prevent the droplet from following the external excitation. As a result, the droplet shape becomes essentially quasi-stationary, i.e., the oscillation will terminate. It is also noted that viscous dissipation is nearly negligible in ACEW due to the small magnitude of the viscous force.

Figure 11 depicts the details of the droplet oscillations in terms of the normalized contact radius, normalized height and contact angle at three resonance frequencies, $f=27 \mathrm{~Hz}, 79 \mathrm{~Hz}$ and $150 \mathrm{~Hz}$. The symbols are data obtained from the experiments and the lines are sinusoidal fit. At all three frequencies, the instants of the maximum contact angle always correspond to the minimum contact radius. Thus, these two parameters are out of phase with a constant phase angle of $90^{\circ}$. In contrast, the phase relation between the drop height and the contact radius seems to be frequency-dependent. For instance, they are out of phase at $27 \mathrm{~Hz}$ and $150 \mathrm{~Hz}$ but in phase at $79 \mathrm{~Hz}$. Furthermore, it is surprising to note that the droplet oscillations closely follow the sinusoidal waveform of the input signals at all three frequencies, although, according to the DCEW results in Fig. 6, the droplet can respond fully to a step stimulus of frequency up to $67 \mathrm{~Hz}$ (with a corresponding actuating frequency $f=33 \mathrm{~Hz}$ ) as it has a time constant of $15 \mathrm{~ms}$. In fact, both observations for DCEW and ACEW suggest the EW-actuated droplet system mimics a second-order dynamic system, such as a driven harmonic oscillator with viscous damping, which follows a parametric equation [56]

$$
\frac{d^{2} x}{d t^{2}}+2 \zeta \omega_{n} \frac{d x}{d t}+\omega_{n}^{2} x=\frac{F(t)}{m}
$$

where $x$ is the measure of the oscillation, $\omega_{n}$ is the resonant angular frequency, $\zeta$ is the damping ratio, $m$ is the effective mass of the system, and $F(t)$ is the external driving force. Interestingly, a few phenomenological models with governing equations of a similar form to Eq. (27) have been developed to describe the droplet motion ([26] [57] [58, 59]). While it remains elusive if the EW-actuated droplet system complies rigorously with Eq. (27), the results in Figs. 6 and 11 indicate that methodologies from the discipline of dynamic systems may prove very useful in understanding the nonlinear oscillating behavior of electrowetting systems in the future work.

Figure 12 illustrates the velocity vector field and the pressure distribution inside and around the droplet obtained from the VOF-CSF simulations. The results correspond to three representative time 
instants in the $\mathrm{P}_{2}, \mathrm{P}_{4}$ and $\mathrm{P}_{6}$ oscillation modes (Note: 1) the initial time instant, $t=0 \mathrm{~ms}$, was chosen somewhat arbitrarily, and 2) the images shown were for the descending half-cycle of the droplet movement). It is interesting to notice that the velocity vector field is in very good agreement with the numerical results obtained in $[32,34]$. Furthermore, recirculation swirls can be identified from the streamline contours, and the number of vortices matches the respective resonance modes. For instance, two vortices can be observed for the $\mathrm{P}_{2}$ mode, four for $\mathrm{P}_{4}$ mode and six for $\mathrm{P}_{6}$ mode. The centers of the vortices are located either at or close to the free surface, which correspond approximately to the nodal points marked in Fig. 8. It is also noticed that, due to the decaying oscillation amplitudes of the droplet, the size of the vortices decreases significantly from the $\mathrm{P}_{2}$ mode to $\mathrm{P}_{6}$ mode.

As discussed for the case of DCEW, the droplet motion originates from the moving contact line and the perturbation subsequently spreads along the interface toward the apex of the droplet. In ACEW, propagating surface waves are generated due to the cyclic forcing mechanism at the contact line. By setting the origin at the center of the droplet, Fig. 13 depicts the instantaneous interfacial shape extracted from the numerical data (Figs. 7 and 12). It is seen that $t=0 \mathrm{~ms}$ marks the moment at which the wave fronts meet at the apex $\left(\phi=90^{\circ}\right)$ and the droplet height reaches the maximum due to the geometric focusing effect. Consequently, the waves are reflected back towards the contact line $\left(\phi=0^{\circ}\right)$ without much damping of the oscillation amplitudes. The nodal points for the $\mathrm{P}_{2}, \mathrm{P}_{4}$ and $\mathrm{P}_{6}$ modes can be identified in Figs. 13 (b)-(d) as the intersection points of the oscillation curves. A standing-wave type of behavior is also observed. Taking the $\mathrm{P}_{6}$ mode as an example, it can be deduced from Figs. 13 (a) and (d) that the radius connecting the nodal point and the origin is $r_{0} \sim 1.6 \mathrm{~mm}$, and the central angle between two adjacent nodal points is $\phi_{0} \sim 32^{\circ}$. Thus, the wavelength of the surface waves can be estimated as the arc length, $\lambda_{s} \sim 2 r_{0} \phi_{0}=1.79 \mathrm{~mm}$. Since the $\mathrm{P}_{6}$ mode corresponds to $f_{n}=318 \mathrm{~Hz}$, the propagation speed of the waves can be calculated as $u_{p}=f_{n} \lambda_{s}=0.57 \mathrm{~m} / \mathrm{s}$, which agrees well with the phase velocity for capillary waves [60].

\section{CONCLUSIONS}

A combined numerical and experimental approach was applied to explore the dynamics of EWinduced droplet motion. The time history of the contact radius, height and instantaneous contact angle of the droplet was investigated for a step input DC signal. The resonance oscillations of the droplet at different actuating frequencies were studied for sinusoidal AC signals. The VOF-CSF method was used to develop CFD models that incorporate an MKT-based dynamic contact angle model. Numerical predictions of various parameters of EW-induced droplet dynamics were validated with the experimental measurements obtained from high-speed photography. This work will help to advance the fundamental 
understanding of droplet dynamics involved in various EW-based systems, especially in the fledging field of digitized active cooling for high-heat-flux thermal management of microsystems.

The key findings can be summarized as follows: (1) A proper selection of the dynamic contact angle model is essential to numerical analysis of droplet dynamics. In this regard, an MKT-based model that considers the contact line friction and the pinning force at the moving contact line was successfully implemented. Special attentions were paid to the acquisition of the contact line velocity and the pinning force, whereas a fitting value was selected for the friction coefficient. (2) The response time of a droplet to external excitations can be characterized from the droplet's expansion and contraction processes under DCEW. (3) When actuated at resonance frequencies in ACEW, the droplet exhibits time-harmonic shape oscillations. The oscillation amplitude is found to decrease with increasing actuating frequency, due to the dominant effect of inertia over other forces. The surface wave propagates with a similar phase velocity for capillary waves. (4) Overall, the dynamics of EW-actuated droplet motion mimics that of a second-order dynamic system.

\section{ACKNOWLEDGMENTS}

The authors are grateful to Professor Suresh V. Garimella at Purdue University for insightful discussions. They acknowledge the financial supports from the National Science Foundation (Grants No. 0927340 and No. 1236606).

\section{REFERENCES}

[1] F. Mugele, J.C. Baret, Electrowetting: From basics to applications, J Phys-Condens Mat, 17(28) (2005) R705R774.

[2] S. Kuiper, B.H.W. Hendriks, Variable-focus liquid lens for miniature cameras, Applied Physics Letters, 85(7) (2004) 1128-1130.

[3] T. Roques-Carmes, R.A. Hayes, L.J.M. Schlangen, A physical model describing the electro-optic behavior of switchable optical elements based on electrowetting, Journal of Applied Physics, 96(11) (2004) 6267-6271.

[4] R.A. Hayes, B.J. Feenstra, Video-speed electronic paper based on electrowetting, Nature, 425(6956) (2003) 383385 .

[5] E. Baird, K. Mohseni, Digitized Heat Transfer: A New Paradigm for Thermal Management of Compact Micro Systems, IEEE Transactions on Components and Packaging Technologies, 31(1) (2008) 143-151.

[6] S.V. Garimella, T. Persoons, J. Weibel, L.-T. Yeh, Technological drivers in data centers and telecom systems: Multiscale thermal, electrical, and energy management, Applied Energy, 107 (2013) 66-80.

[7] R.S. Hale, V. Bahadur, Electrowetting Heat Pipes for Heat Transport Over Extended Distances, IEEE Transactions on Components, Packaging and Manufacturing Technology, 5(10) (2015) 1441-1450.

[8] G. Cha, C.-J. Kim, Y.S. Ju, Thermal conductance switching based on the actuation of liquid droplets through the electrowetting on dielectric (EWOD) phenomenon, Applied Thermal Engineering, 98 (2016) 189-195.

[9] K.H. Kang, How electrostatic fields change contact angle in electrowetting, Langmuir, 18(26) (2002) 1031810322 .

[10] M. Paneru, C. Priest, R. Sedev, J. Ralston, Static and Dynamic Electrowetting of an Ionic Liquid in a Solid/Liquid/Liquid System, Journal of the American Chemical Society, 132(24) (2010) 8301-8308.

[11] L. Chen, E. Bonaccurso, Electrowetting -- from statics to dynamics, Advances in colloid and interface science, $210(2014) 2-12$.

[12] R.L. Hoffman, A study of the advancing interface. I. Interface shape in liquid—gas systems, Journal of Colloid and Interface Science, 50(2) (1975) 228-241. 
[13] E.B. Dussan, On the Spreading of Liquids on Solid Surfaces: Static and Dynamic Contact Lines, Annual Review of Fluid Mechanics, 11(1) (1979) 371-400.

[14] C. Decamps, J. De Coninck, Dynamics of spontaneous spreading under electrowetting conditions, Langmuir, 16(26) (2000) 10150-10153.

[15] T.D. Blake, A. Clarke, E.H. Stattersfield, An Investigation of Electrostatic Assist in Dynamic Wetting, Langmuir, 16(6) (2000) 2928-2935.

[16] R.G. Cox, The Dynamics of the Spreading of Liquids on a Solid-Surface, I. Viscous Flow, Journal of Fluid Mechanics, 168 (1986) 169-194.

[17] R.G. Cox, Inertial and viscous effects on dynamic contact angles, Journal of Fluid Mechanics, 357 (1998) 249278.

[18] T.D. Blake, J.M. Haynes, Kinetics of liquid/liquid displacement, Journal of Colloid and Interface Science, 30(3) (1969) 421-423.

[19] T.D. Blake, The physics of moving wetting lines, Journal of Colloid and Interface Science, 299(1) (2006) 1-13.

[20] S.R. Ranabothu, C. Karnezis, L.L. Dai, Dynamic wetting: Hydrodynamic or molecular-kinetic?, Journal of Colloid and Interface Science, 288(1) (2005) 213-221.

[21] S.R. Annapragada, J.Y. Murthy, S.V. Garimella, Prediction of droplet dynamics on an incline, International Journal of Heat and Mass Transfer, 55(5-6) (2012) 1466-1474.

[22] X.D. Wang, X.F. Peng, D.Z. Lee, Dynamic wetting and stress singularity on contact line, Science in China Series E-Technological Sciences, 46(4) (2003) 407-417.

[23] J.M. Oh, S.H. Ko, K.H. Kang, Analysis of electrowetting-driven spreading of a drop in air, Physics of Fluids, 22(3) (2010).

[24] K.L. Wang, T.B. Jones, Electrowetting dynamics of microfluidic actuation, Langmuir, 21(9) (2005) 4211-4217.

[25] Z. Keshavarz-Motamed, L. Kadem, A. Dolatabadi, Effects of dynamic contact angle on numerical modeling of electrowetting in parallel plate microchannels, Microfluidics and Nanofluidics, 8(1) (2010) 47-56.

[26] S.R. Annapragada, S. Dash, S.V. Garimella, J.Y. Murthy, Dynamics of Droplet Motion under Electrowetting Actuation, Langmuir, 27(13) (2011) 8198-8204.

[27] J. Hong, Y.K. Kim, K.H. Kang, J.M. Oh, I.S. Kang, Effects of Drop Size and Viscosity on Spreading Dynamics in DC Electrowetting, Langmuir, 29(29) (2013) 9118-9125.

[28] S.J. Hong, J. Hong, H.W. Seo, S.J. Lee, S.K. Chung, Fast Electrically Driven Capillary Rise Using Overdrive Voltage, Langmuir, 31(51) (2015) 13718-13724.

[29] J.M. Oh, S.H. Ko, K.H. Kang, Shape Oscillation of a Drop in ac Electrowetting, Langmuir, 24(15) (2008) 8379-8386.

[30] J. Hong, Y.K. Kim, K.H. Kang, J. Kim, S.J. Lee, Effects of drop viscosity on oscillation dynamics induced by AC electrowetting, Sensors and Actuators B-Chemical, 190 (2014) 48-54.

[31] F. Mugele, J.-C. Baret, D. Steinhauser, Microfluidic mixing through electrowetting-induced droplet oscillations, Applied Physics Letters, 88(20) (2006) 204106.

[32] K. Ashoke Raman, R.K. Jaiman, T.-S. Lee, H.-T. Low, A numerical study on electrowetting-induced jumping and transport of droplet, International Journal of Heat and Mass Transfer, 99 (2016) 805-821.

[33] J.S. Hong, S.H. Ko, K.H. Kang, I.S. Kang, A numerical investigation on AC electrowetting of a droplet, Microfluidics and Nanofluidics, 5(2) (2007) 263-271.

[34] Z. Li, Z.W. Zhou, G.H. Hu, Dissipative Particle Dynamics Simulation of Droplet Oscillations in AC Electrowetting, Journal of Adhesion Science and Technology, 26(12-17) (2012) 1883-1895.

[35] X.L. Li, G.W. He, X. Zhang, Numerical simulation of drop oscillation in AC electrowetting, Science ChinaPhysics Mechanics \& Astronomy, 56(2) (2013) 383-394.

[36] R.G. Cox, The dynamics of the spreading of liquids on a solid surface. Part 1. Viscous flow, Journal of Fluid Mechanics, 168 (1986) 169-194.

[37] M. Schneemilch, R.A. Hayes, J.G. Petrov, J. Ralston, Dynamic Wetting and Dewetting of a Low-Energy Surface by Pure Liquids, Langmuir, 14(24) (1998) 7047-7051.

[38] O.V. Voinov, Hydrodynamics of wetting, Fluid Dynamics, 11(5) 714-721.

[39] L.H. Tanner, The spreading of silicone oil drops on horizontal surfaces, Journal of Physics D: Applied Physics, 12(9) (1979) 1473.

[40] S. Glasstone, Laidler, K. J., Eyring, H. J., The Theory of Rate Processes, McGraw-Hill, New York, 1941.

[41] J.I. Frenkel, Kinetic Theory of Liquids, Oxford University Press, Oxford, 1946.

[42] S.W. Walker, B. Shapiro, R.H. Nochetto, Electrowetting with contact line pinning: Computational modeling and comparisons with experiments, Physics of Fluids, 21(10) (2009) 102103. 
[43] E. Atefi, J.A. Mann, Jr., H. Tavana, A Robust Polynomial Fitting Approach for Contact Angle Measurements, Langmuir, 29(19) (2013) 5677-5688.

[44] S.F. Lunkad, V.V. Buwa, K.D.P. Nigam, Numerical simulations of drop impact and spreading on horizontal and inclined surfaces, Chem. Eng. Sci., 62(24) (2007) 7214-7224.

[45] K. Yokoi, D. Vadillo, J. Hinch, I. Hutchings, Numerical studies of the influence of the dynamic contact angle on a droplet impacting on a dry surface, Physics of Fluids, 21(7) (2009).

[46] C.W. Hirt, B.D. Nichols, Volume of fluid (VOF) method for the dynamics of free boundaries, Journal of Computational Physics, 39(1) (1981) 201-225.

[47] M. Bussmann, J. Mostaghimi, S. Chandra, On a three-dimensional volume tracking model of droplet impact, Physics of Fluids, 11(6) (1999) 1406-1417.

[48] S. Sikalo, C. Tropea, E.N. Ganic, Dynamic wetting angle of a spreading droplet, Experimental Thermal and Fluid Science, 29(7) (2005) 795-802.

[49] J.U. Brackbill, D.B. Kothe, C. Zemach, A Continuum Method for Modeling Surface-Tension, Journal of Computational Physics, 100(2) (1992) 335-354.

[50] I.V. Roisman, L. Opfer, C. Tropea, M. Raessi, J. Mostaghimi, S. Chandra, Drop impact onto a dry surface: Role of the dynamic contact angle, Colloids and Surfaces a-Physicochemical and Engineering Aspects, 322(1-3) (2008) 183-191.

[51] M. Renardy, Y. Renardy, J. Li, Numerical Simulation of Moving Contact Line Problems Using a Volume-ofFluid Method, Journal of Computational Physics, 171(1) (2001) 243-263.

[52] S. Afkhami, S. Zaleski, M. Bussmann, A mesh-dependent model for applying dynamic contact angles to VOF simulations, Journal of Computational Physics, 228(15) (2009) 5370-5389.

[53] S. Afkhami, M. Bussmann, Height functions for applying contact angles to 3D VOF simulations, International Journal for Numerical Methods in Fluids, 61(8) (2009) 827-847.

[54] B.A. Nichita, Zun, I., Thome, J. R., A VOF method coupled with a dynamic contact angle model for simulation of two-phase flows with partial wetting, in: 7th International Conference on Multiphase Flow, Tampa, Florida, 2010.

[55] H. Lamb, Hydrodynamics, Cambridge University Press, New York, 1932.

[56] K. Ogata, System Dynamics, Pearson, 2003.

[57] S. Dash, N. Kumari, S.V. Garimella, Frequency-dependent transient response of an oscillating electrically actuated droplet, Journal of Micromechanics and Microengineering, 22(7) (2012).

[58] J.-C. Baret, M.M.J. Decré, F. Mugele, Self-Excited Drop Oscillations in Electrowetting, Langmuir, 23(9) (2007) 5173-5179.

[59] P. Sen, C.-J.C. Kim, Capillary Spreading Dynamics of Electrowetted Sessile Droplets in Air, Langmuir, 25(8) (2009) 4302-4305.

[60] J.M. Oh, D. Legendre, F. Mugele, Shaken not stirred - On internal flow patterns in oscillating sessile drops, EPL, 98(3) (2012). 
Table 1. Comparison of the actuating frequency, the oscillating frequency and the resonant frequency of the droplet.

\begin{tabular}{cccc}
\hline Mode & $\begin{array}{c}\text { Actuating frequency } \\
f(\mathrm{~Hz})\end{array}$ & $\begin{array}{c}\text { Oscillating frequency } \\
\mathrm{f}_{\mathrm{exp}}(\mathrm{Hz})\end{array}$ & $\begin{array}{c}\text { Resonant frequency } \\
f_{n}(\mathrm{~Hz})\end{array}$ \\
\hline $\mathrm{P}_{2}$ & 27 & 50 & 58 \\
\hline $\mathrm{P}_{4}$ & 79 & 156 & 174 \\
\hline $\mathrm{P}_{6}$ & 150 & 278 & 318 \\
\hline
\end{tabular}




\section{Tables of figures}

Figure 1. Experimental setup for EW tests.

Figure 2. Computational domain and the boundary conditions.

Figure 3. Comparison of the predicted time evolution of the normalized droplet contact radius vs. the experimental data for a step voltage $(\mathrm{V}=32 \mathrm{~V})$.

Figure 4. Comparison of the predicted instantaneous droplet shape with experiments for a step voltage $(\mathrm{V}=32 \mathrm{~V})$.

Figure 5. Internal flow field and pressure distribution within the droplet for a step voltage $(\mathrm{V}=$ $32 \mathrm{~V})$.

Figure 6 . Time history of the normalized contact radius, normalized height and contact angle of the droplet for a pulse wave voltage $\left(\mathrm{V}=32 \mathrm{~V}\right.$ and $\left.T_{0}=500 \mathrm{~ms}\right)$.

Figure 7. Comparison of the predicted instantaneous shape oscillations of the droplet with experiments during one oscillation cycle at $32 \mathrm{~V}_{\mathrm{RMS}}$.

Figure 8. Resonant modes of shape oscillation of the droplet.

Figure 9. Oscillation amplitudes as a function of actuating frequency.

Figure 10. Comparison of the electrical force, inertia, capillary force, contact line friction force and viscous force.

Figure 11. Droplet oscillation at resonance frequencies: (a) $f=27 \mathrm{~Hz}$, (b) $f=79 \mathrm{~Hz}$ and (c) $f=$ $150 \mathrm{~Hz}$.

Figure 12. Velocity vector field, pressure distribution and vortex zone inside and around the droplet at resonance modes (Note: Pressure is relative to the ambient).

Figure 13. Instantaneous interfacial shapes of the droplet. (a) Schematic of the oscillating droplet, (b) $\mathrm{P}_{2}$ mode $(f=27 \mathrm{~Hz}),(\mathrm{c}) \mathrm{P}_{4}$ mode $(f=79 \mathrm{~Hz})$, and (d) $\mathrm{P}_{6}$ mode $(f=150 \mathrm{~Hz})$. 
Arbitrary waveform generator

Computer

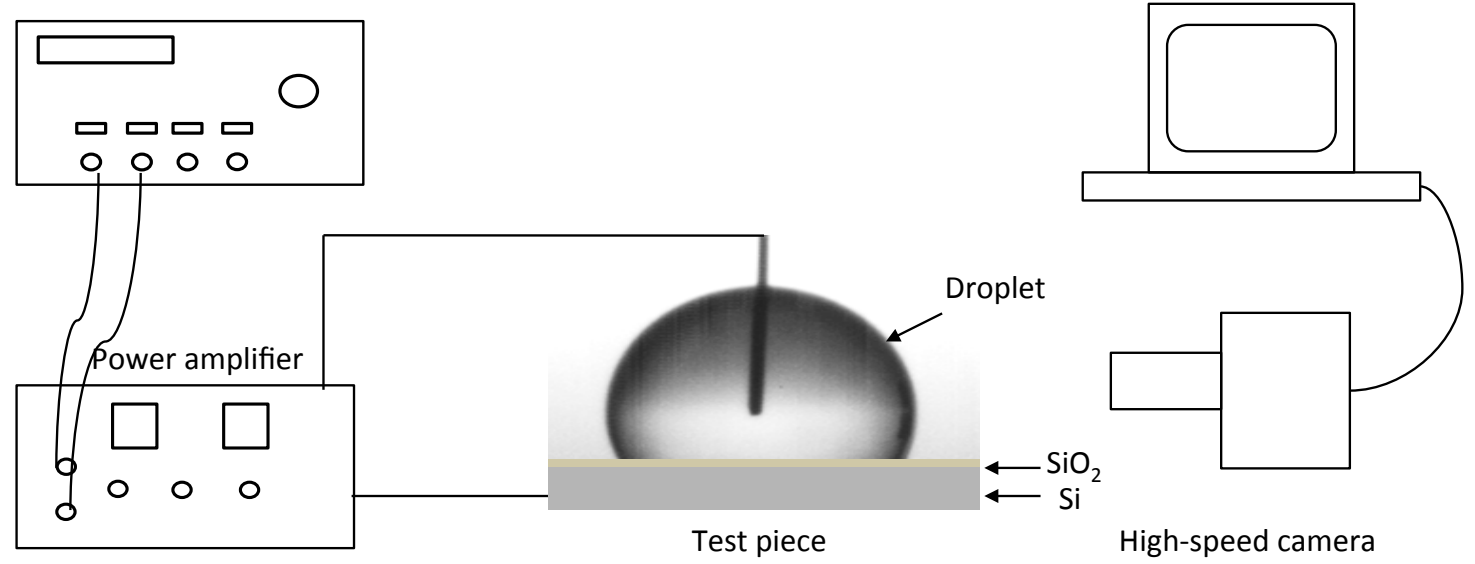

Figure 1. Experimental setup for EW tests. 


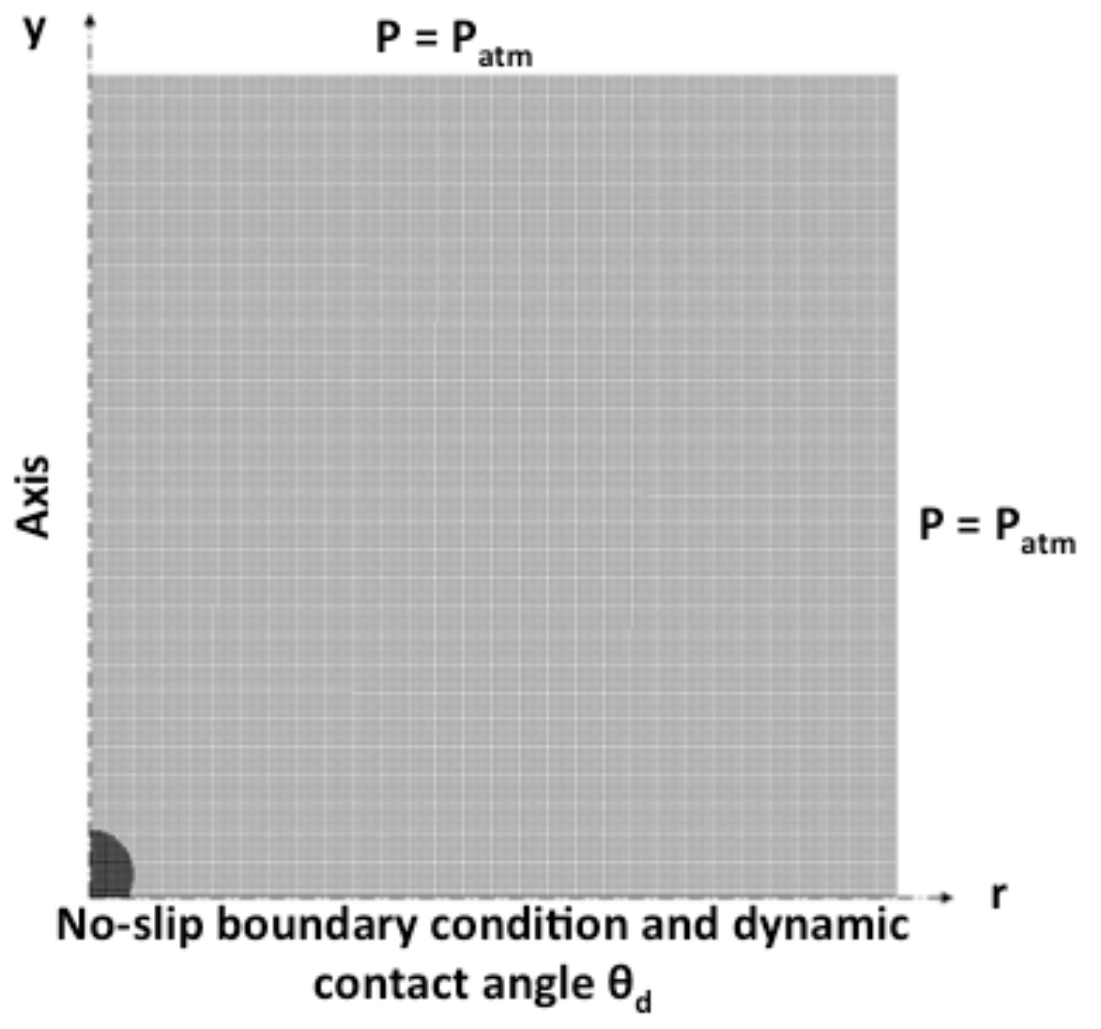

Figure 2. Computational domain and the boundary conditions. 


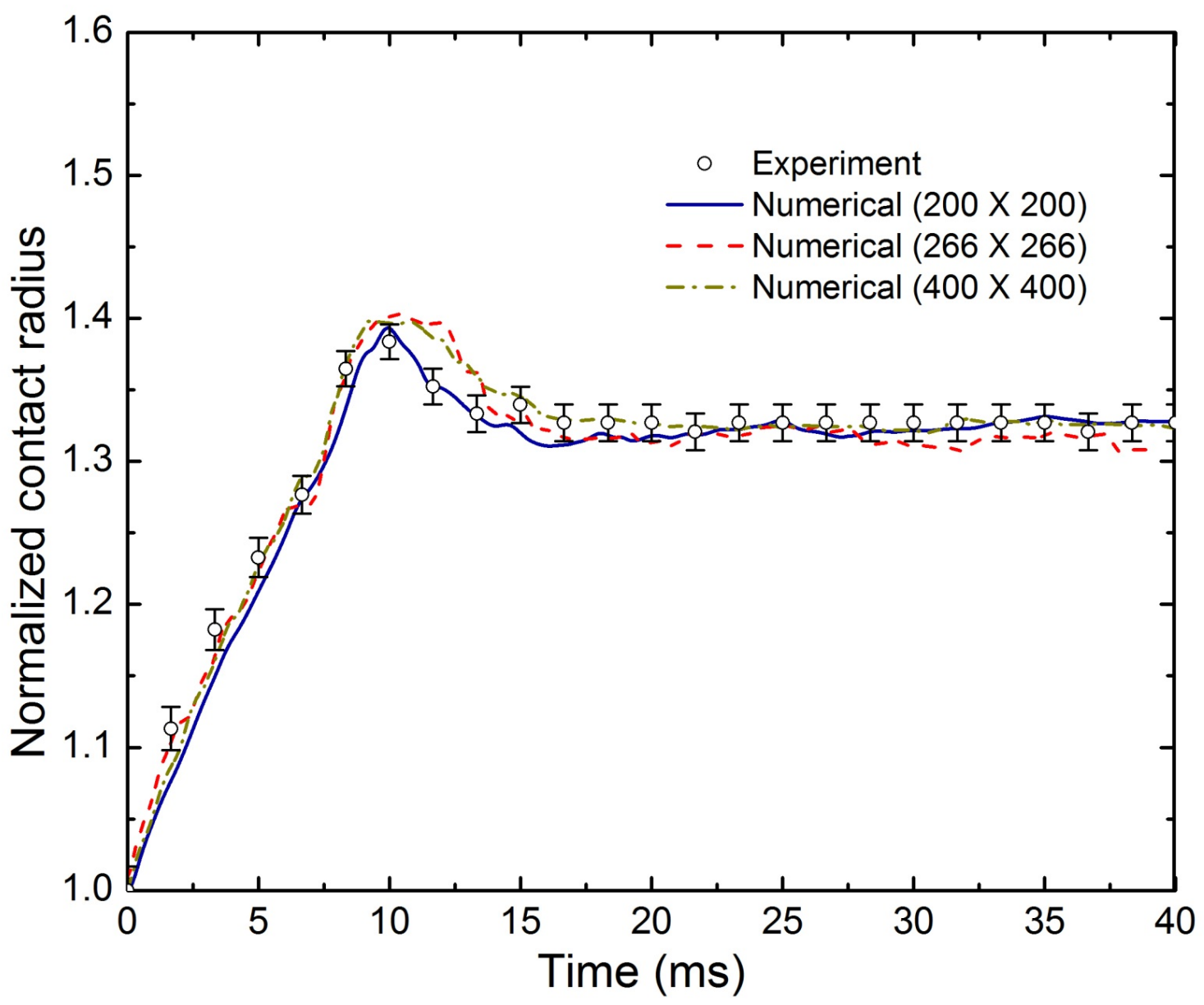

Figure 3. Comparison of the predicted time evolution of the normalized droplet contact radius vs. the experimental data for a step voltage $(\mathrm{V}=32 \mathrm{~V})$. 
คิดคดล 


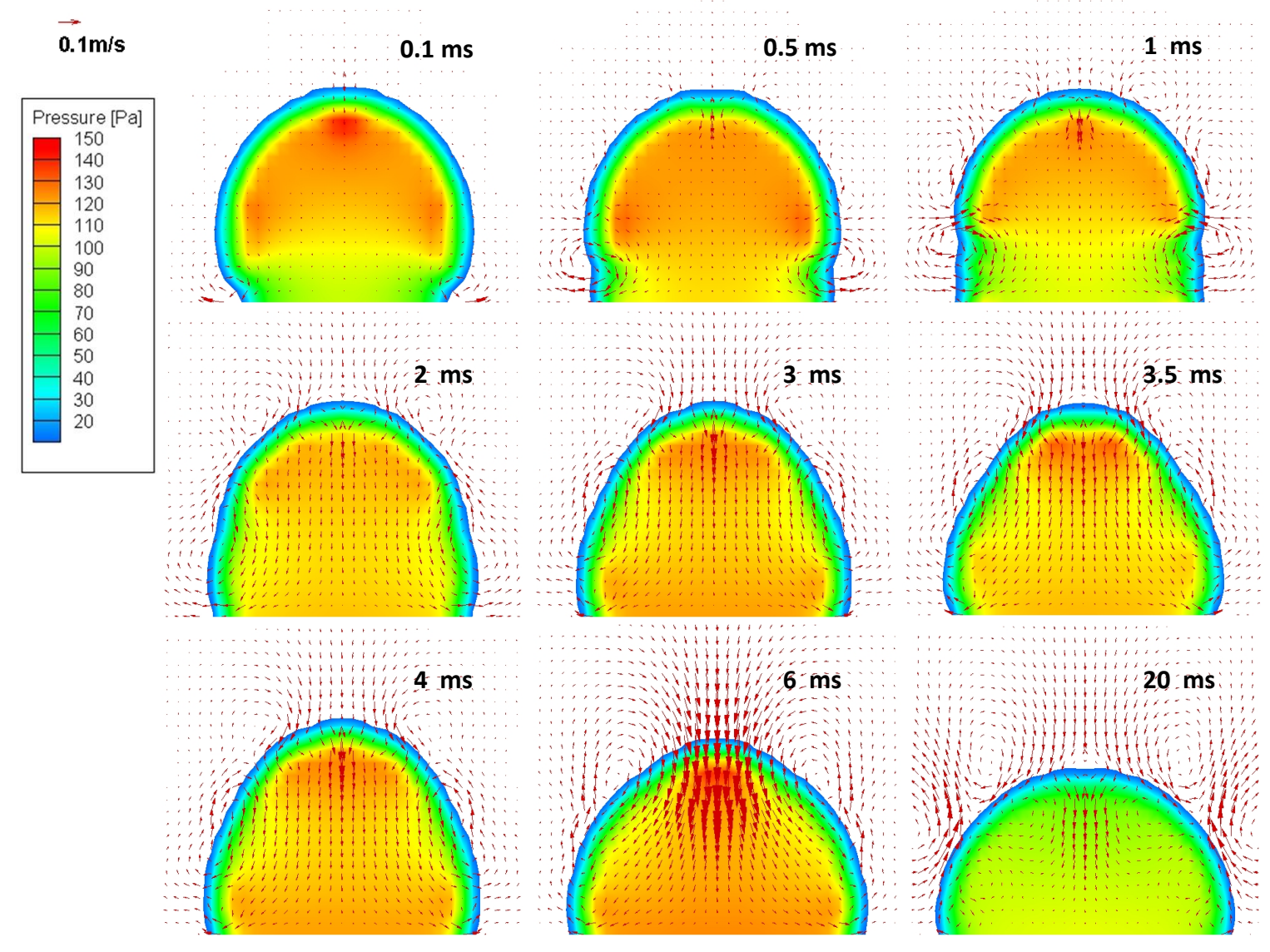

Figure 5. Internal flow field within the droplet for a step voltage $(\mathrm{V}=32 \mathrm{~V})$. 


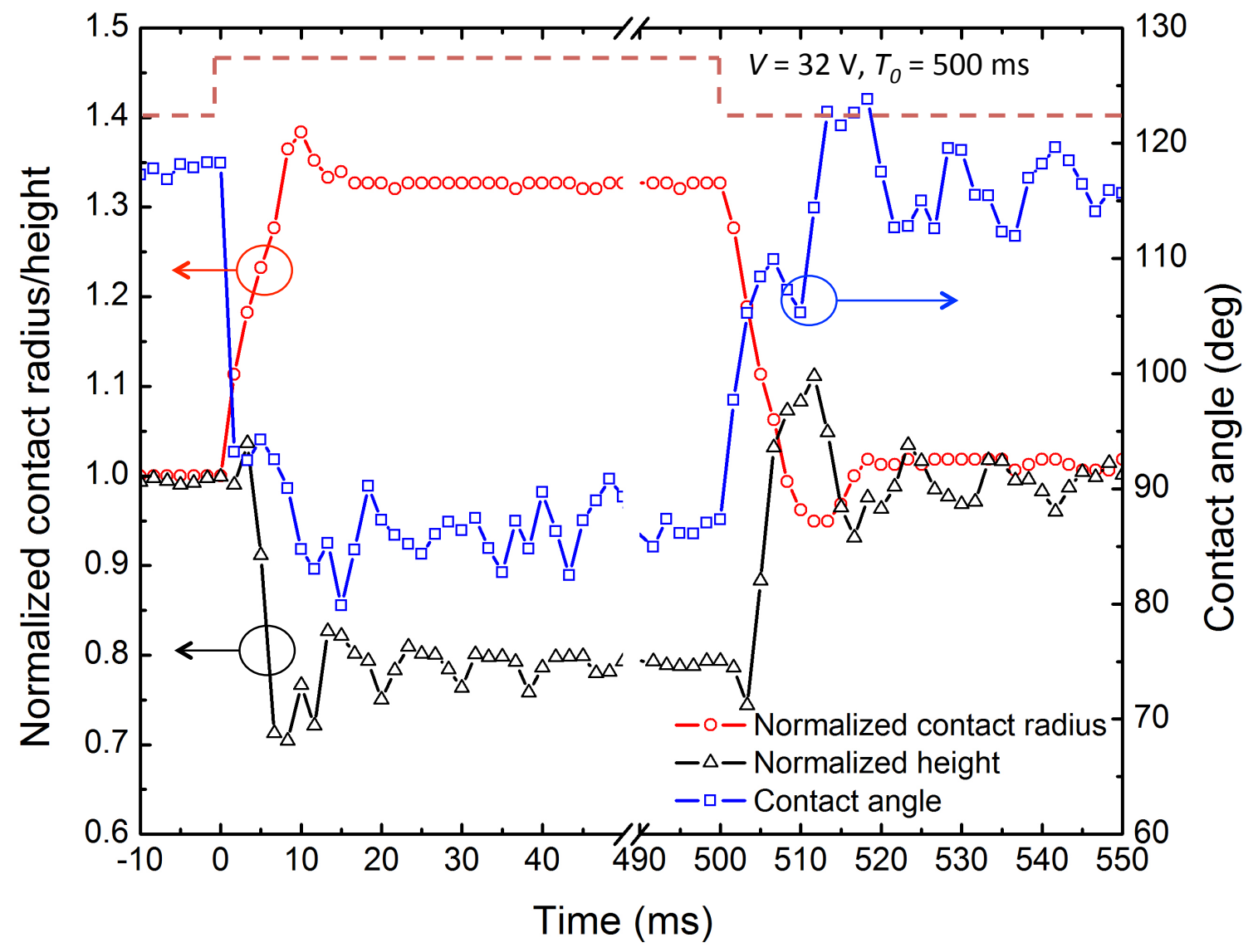

Figure 6. Time history of the normalized contact radius, normalized height and contact angle of the droplet for a pulse wave voltage $\left(\mathrm{V}=32 \mathrm{~V}\right.$ and $\left.T_{0}=500 \mathrm{~ms}\right)$. 


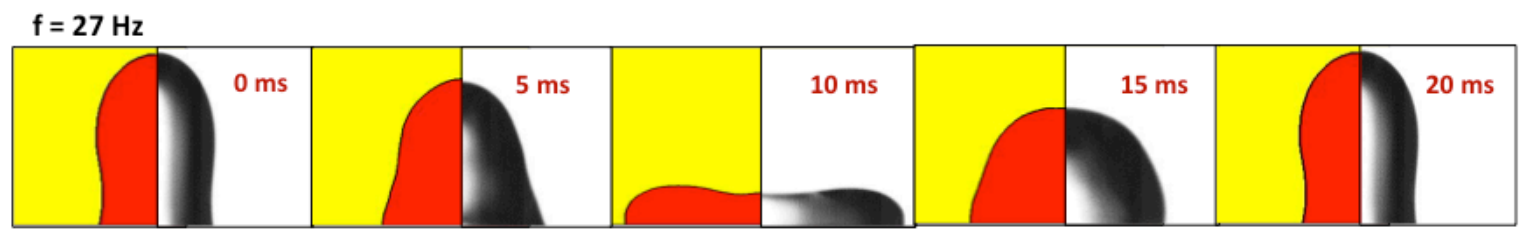

$f=79 \mathrm{~Hz}$
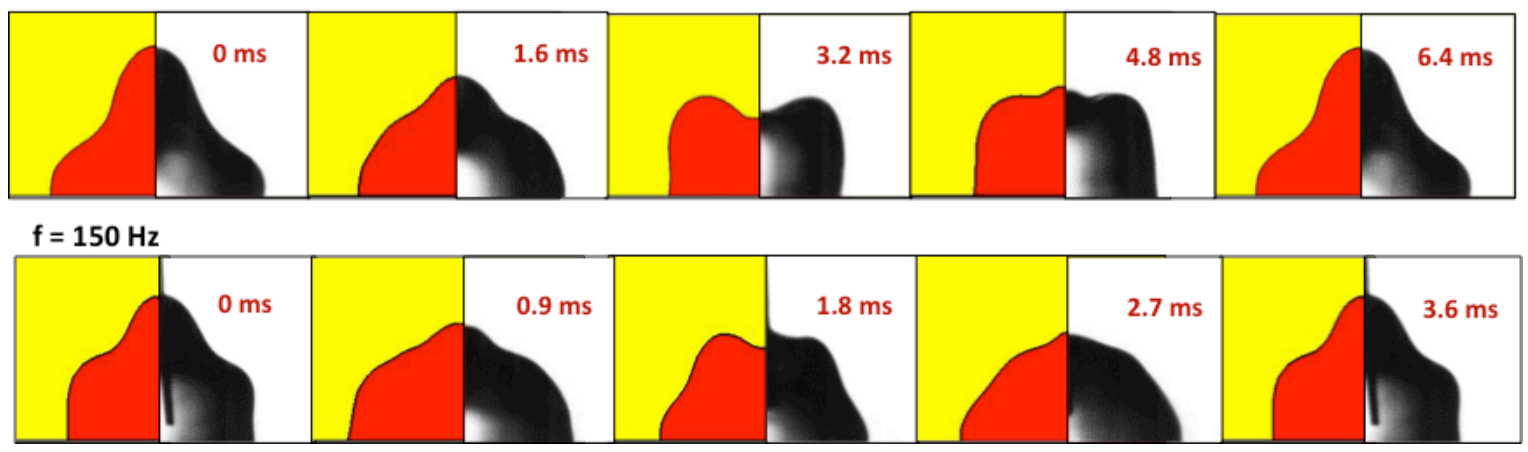

Figure 7. Comparison of the predicted instantaneous shape oscillations of the droplet (left) with experiments (right) during one oscillation cycle at $36 \mathrm{~V}_{\mathrm{RMS}}$. 

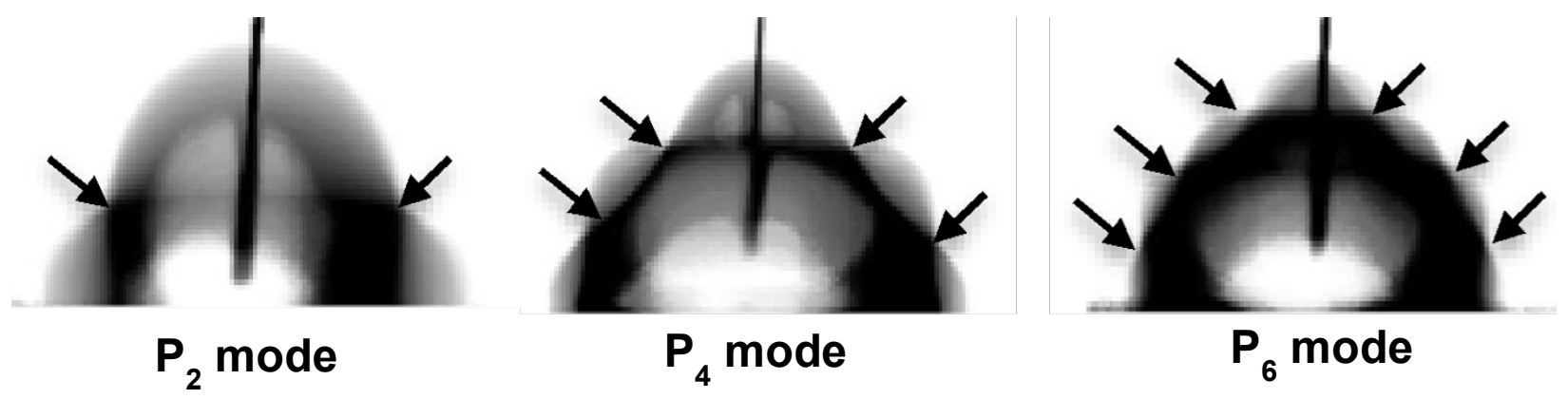

Figure 8 . Resonance modes of shape oscillations of the droplet. 


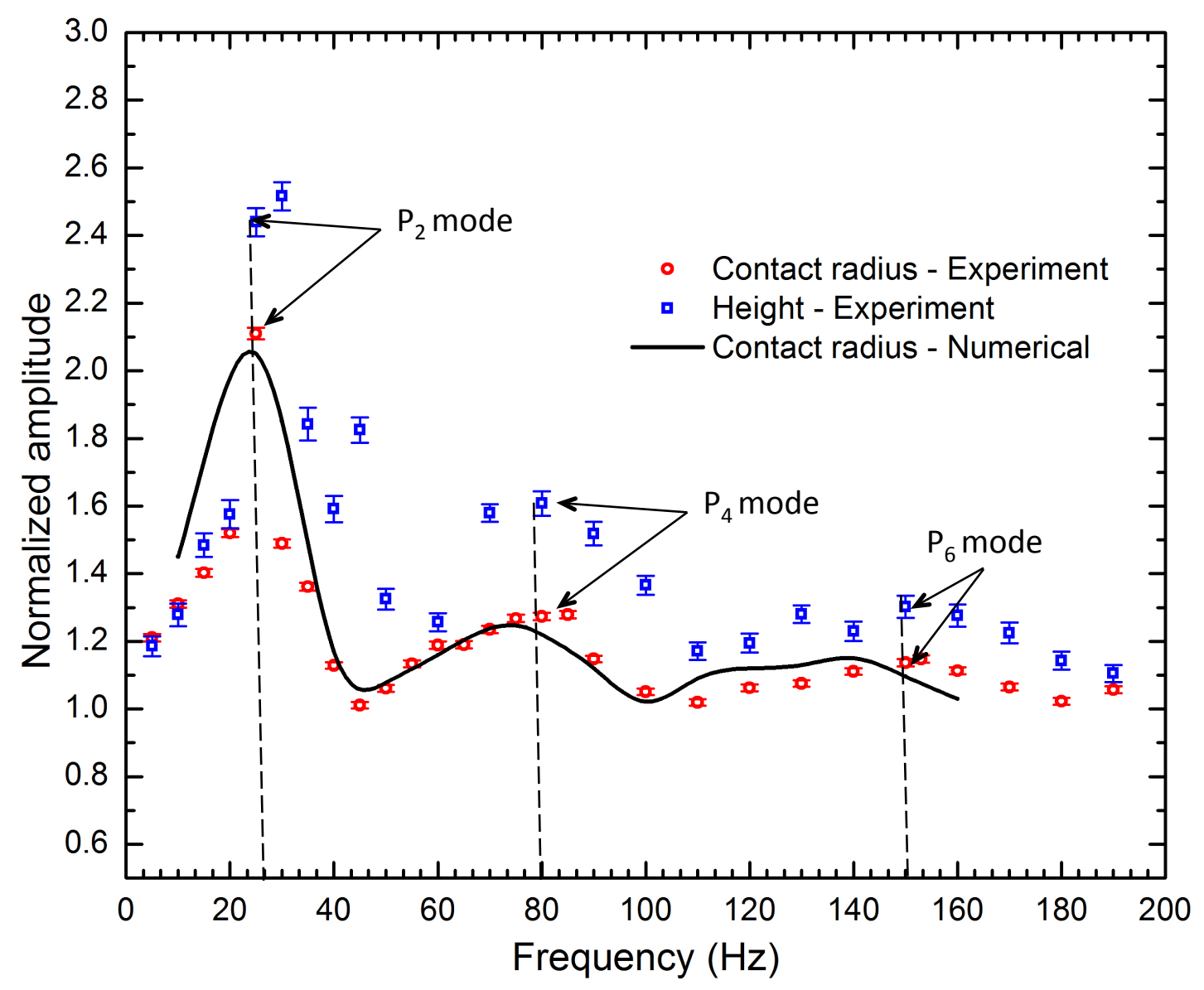

Figure 9. Oscillation amplitudes as a function of actuating frequency. 


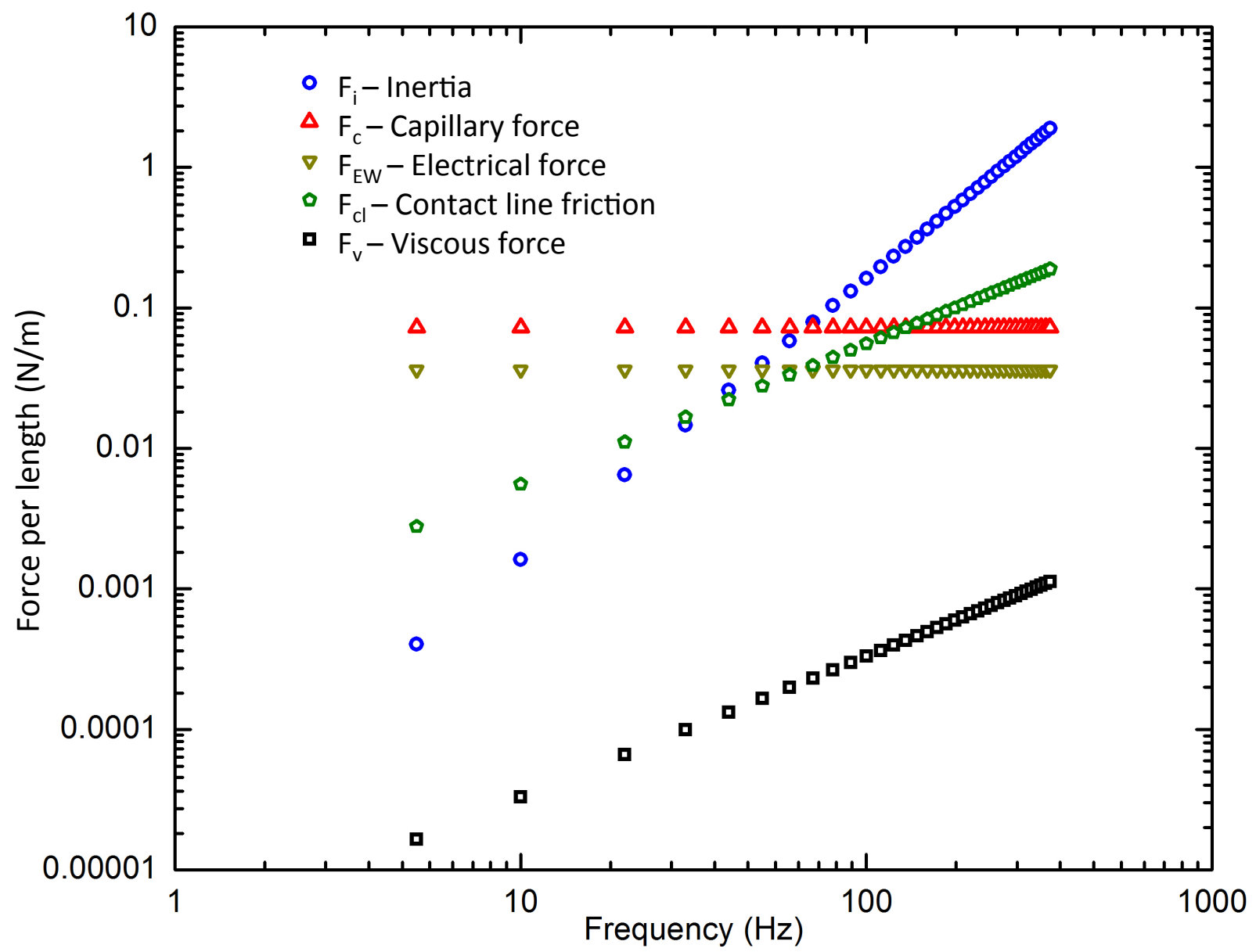

Figure 10. Comparison of the electrical force, inertia, capillary force, contact line friction force and viscous force. 


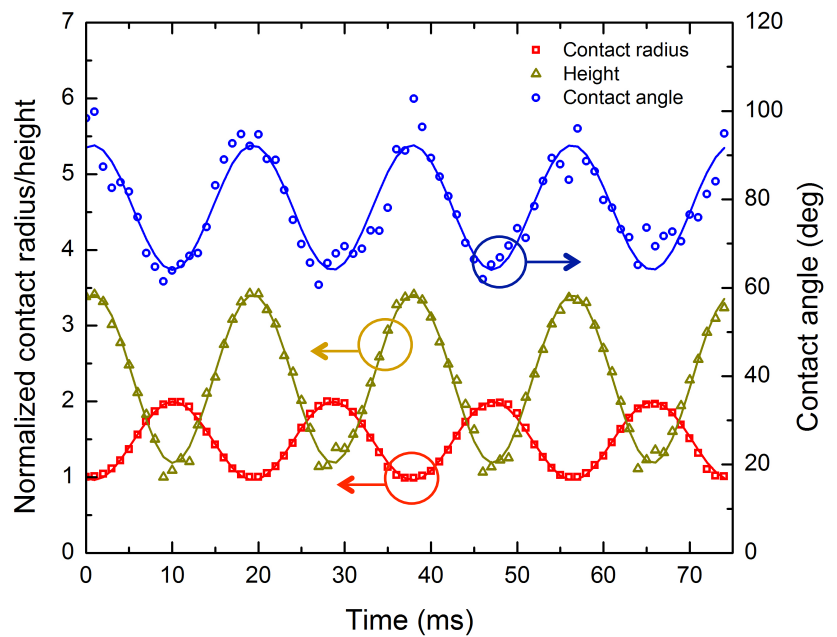

(a)

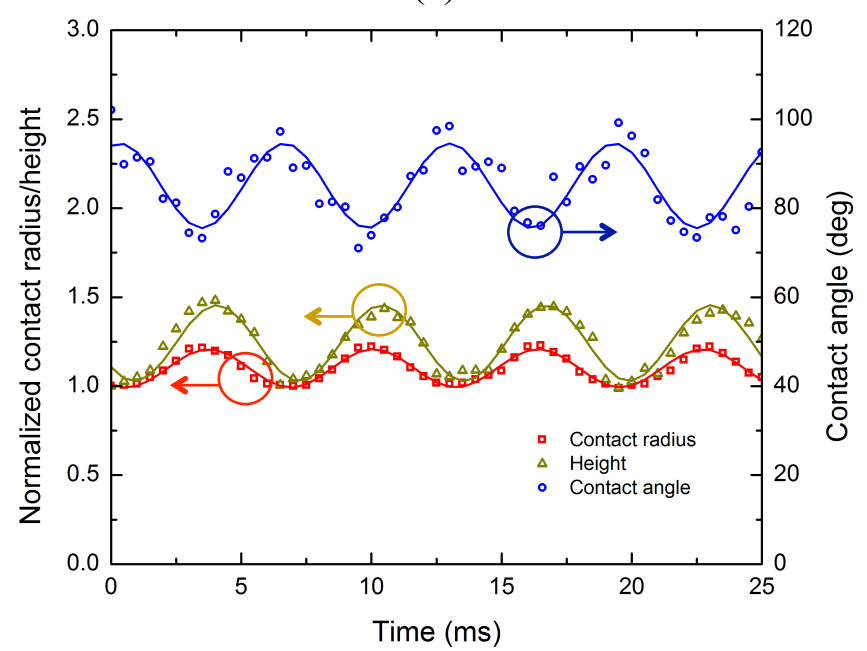

(b)

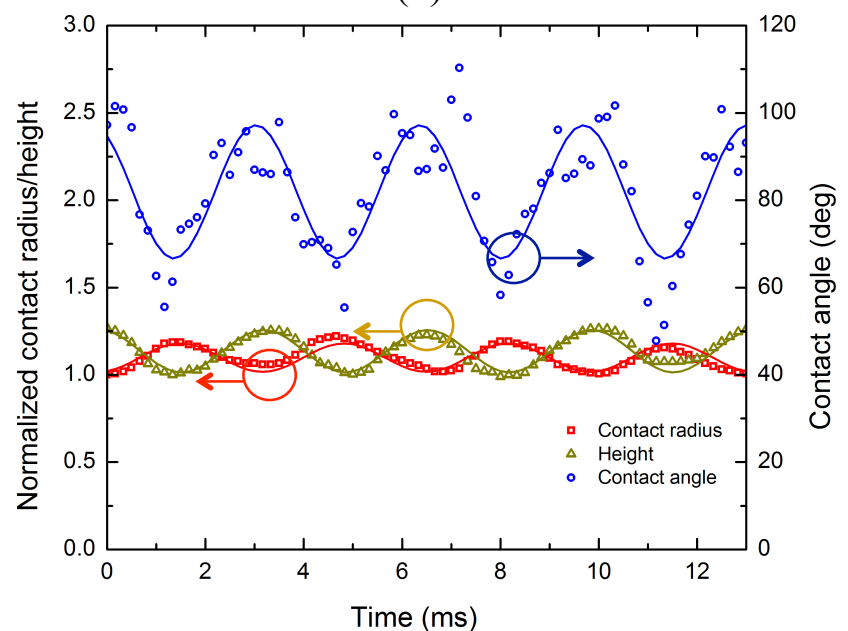

(c)

Figure 11. Droplet oscillation at resonance frequencies: (a) $f=27 \mathrm{~Hz}$, (b) $f=79 \mathrm{~Hz}$ and (c) $f=150 \mathrm{~Hz}$. 

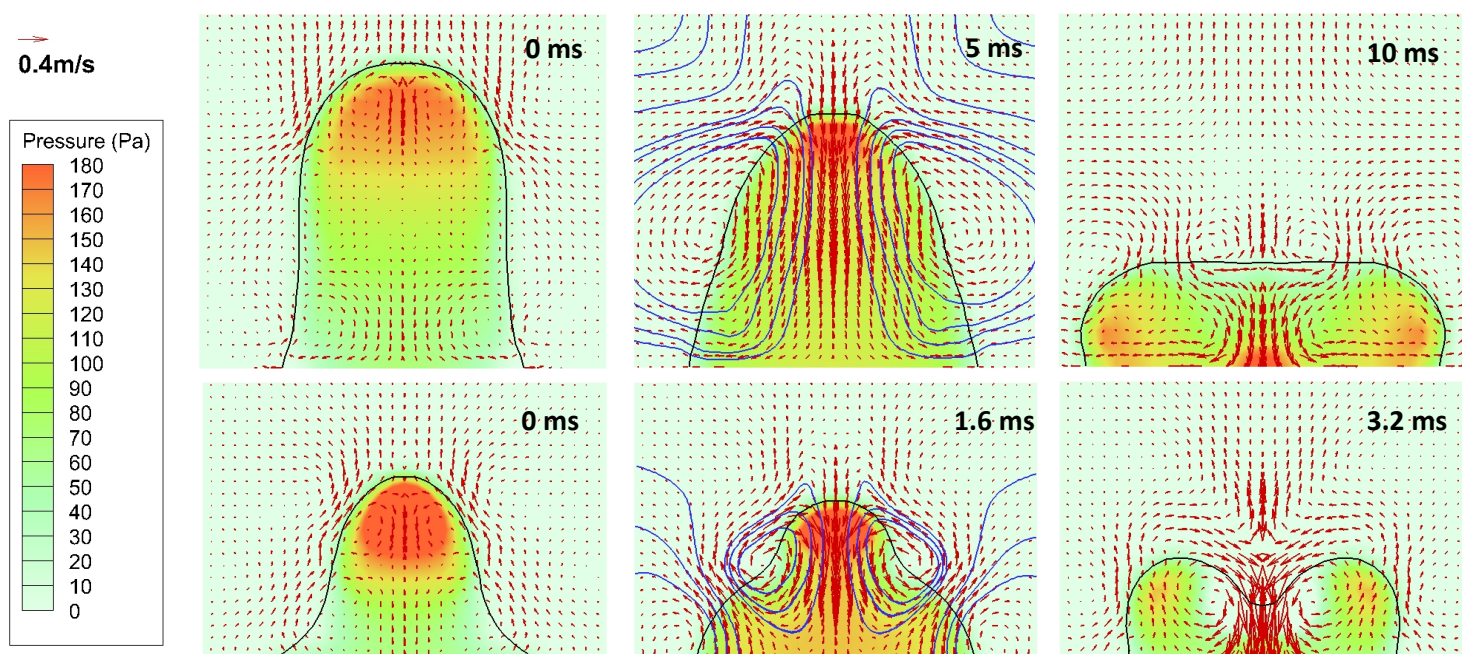

$P_{2}$ mode
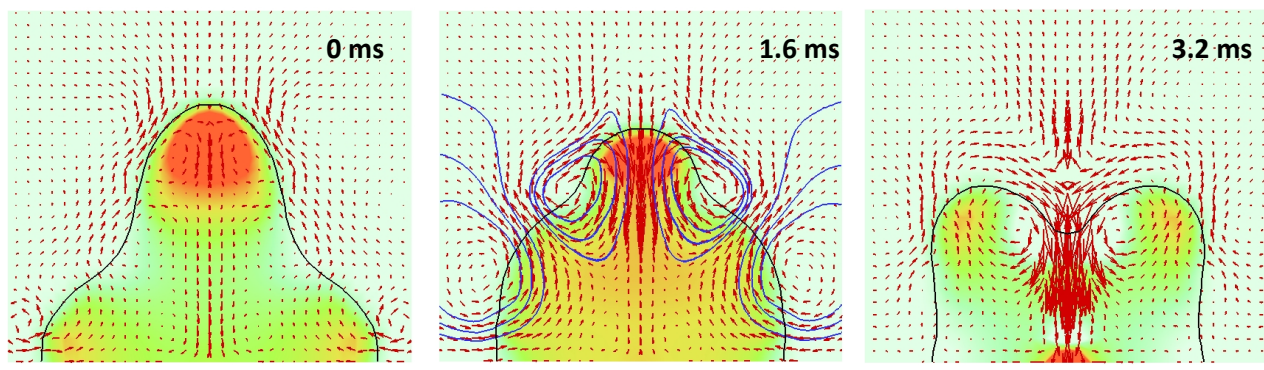

$P_{4}$ mode
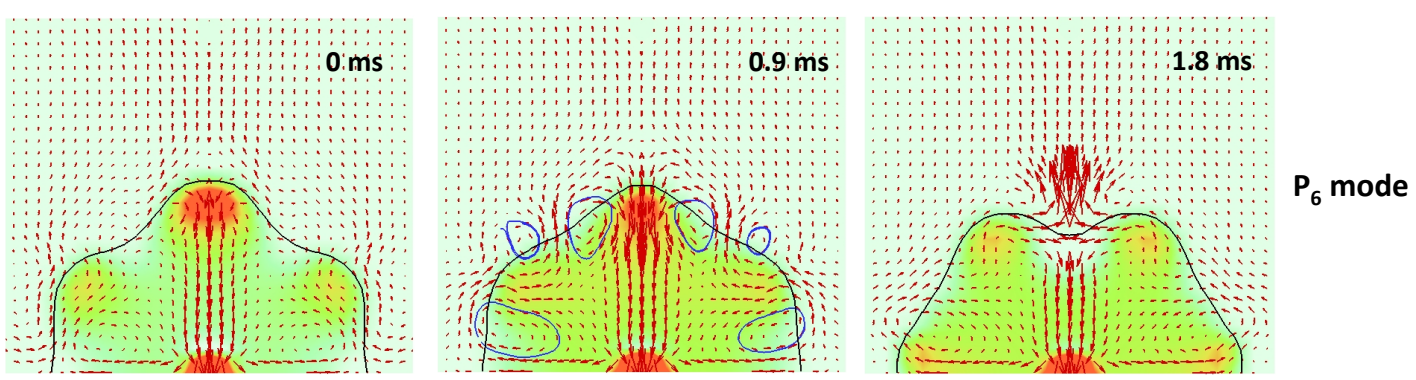

Figure 12. Velocity vector field, pressure distribution and vortex zone inside and around the droplet at resonance modes (Note: Pressure is relative to the ambient). 


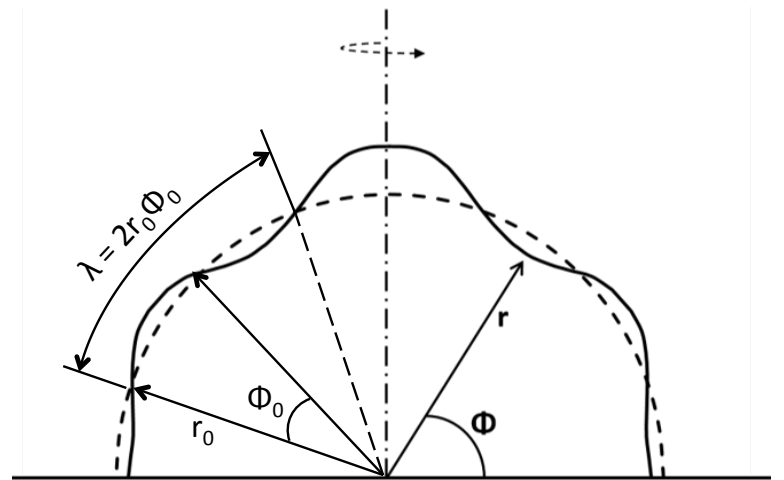

(a)

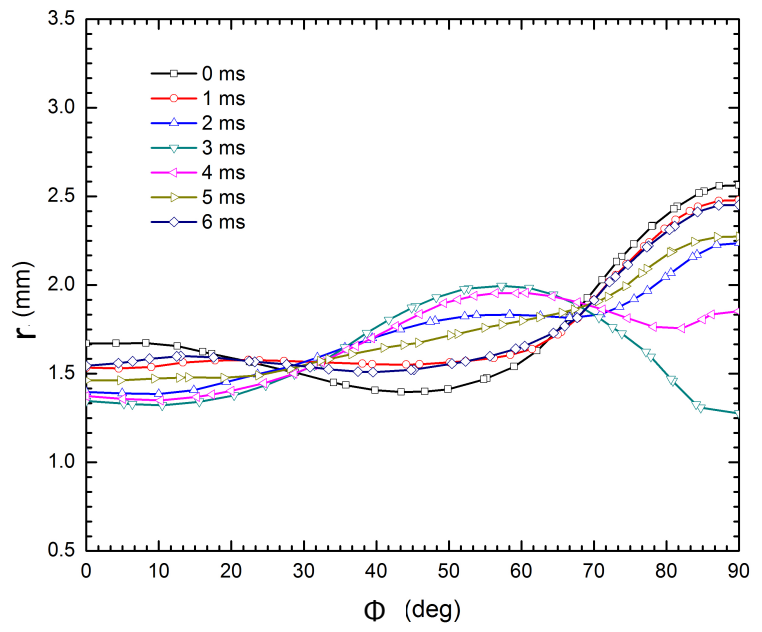

(c)

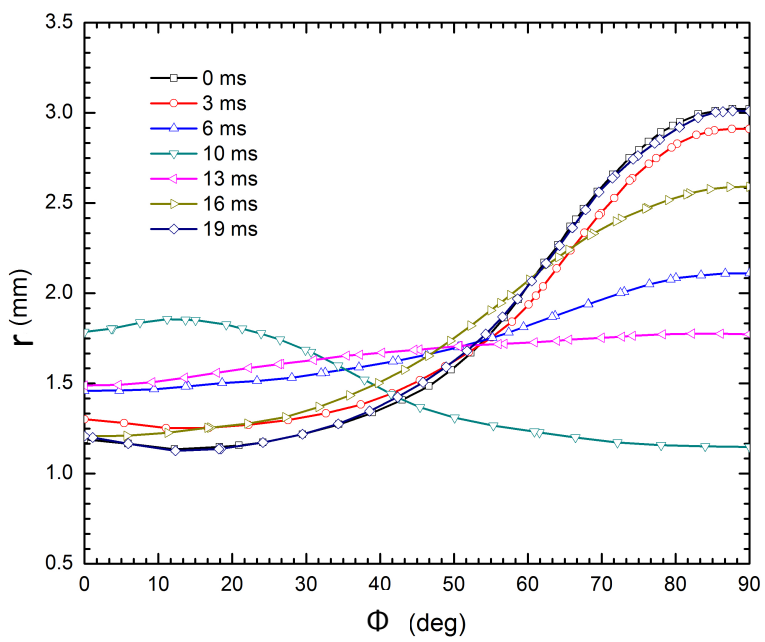

(b)

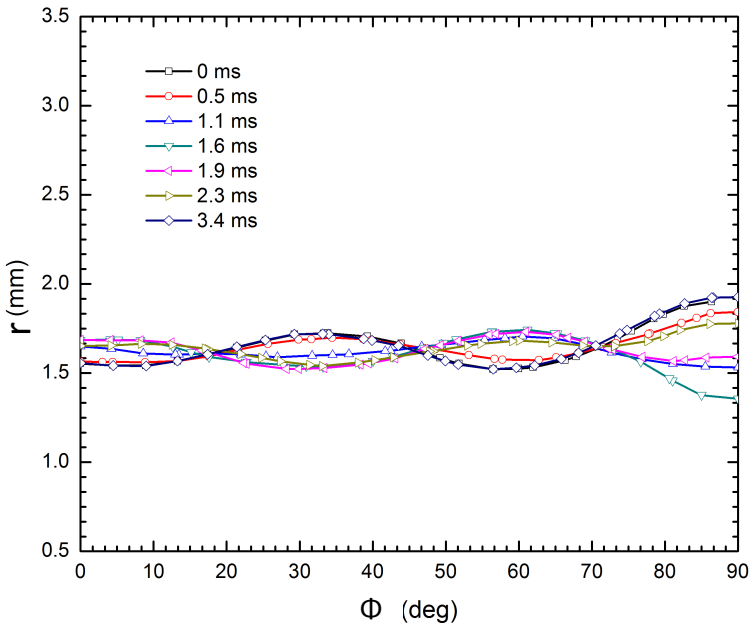

(d)

Figure 13. Instantaneous interfacial shapes of the droplet. (a) Schematic of the oscillating droplet, (b) $\mathrm{P}_{2}$ mode $(f=27 \mathrm{~Hz})$, (c) $\mathrm{P}_{4}$ mode $(f=79 \mathrm{~Hz})$, and (d) $\mathrm{P}_{6}$ mode $(f=150 \mathrm{~Hz})$. 